\title{
Phosphoprotein Phosphatase 1 Isoforms Alpha and Gamma Respond Differently to Prodigiosin Treatment and Present Alternative Kinase Targets in Melanoma Cells
}

\author{
Margarida Fardilha1 ${ }^{*}$, João Figueiredo', Margarita Espona-Fiedler ${ }^{2}$, Juliana Felgueiras ${ }^{1}$, \\ Luís Korrodi-Gregório ${ }^{1}$, Sara L. C. Esteves' ${ }^{1}$, Sandra Rebelo ${ }^{3}$, Odete A. B. da Cruz Silva ${ }^{3}$, \\ Edgar da Cruz e Silva1, Ricardo Pérez-Tómas ${ }^{2}$

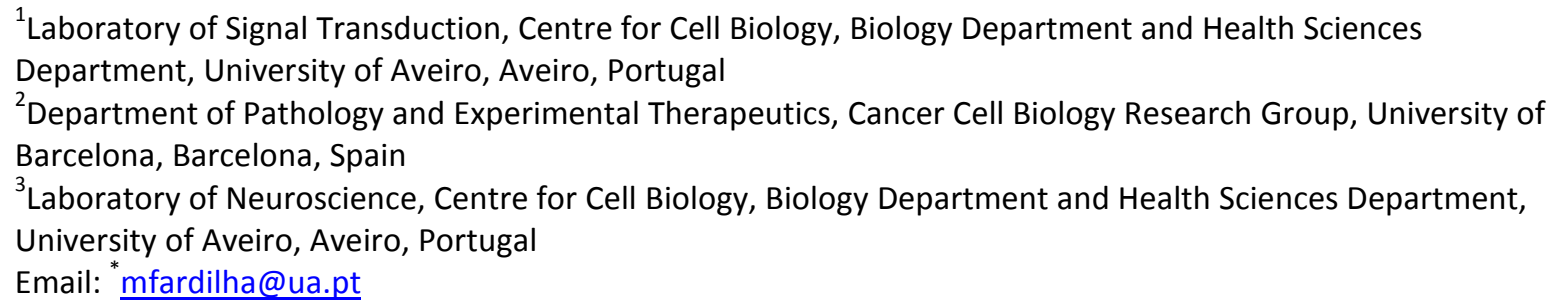

Received 17 March 2014; revised 17 April 2014; accepted 24 April 2014

Copyright (C) 2014 by authors and Scientific Research Publishing Inc.

This work is licensed under the Creative Commons Attribution International License (CC BY). http://creativecommons.org/licenses/by/4.0/

c) (i) Open Access

\section{Abstract}

Reversible protein phosphorylation is a central regulatory mechanism of cell function. Deregulation of the balanced actions of protein kinases and phosphatases has been frequently associated with several pathological conditions, including cancer. Many studies have already addressed the role of protein kinases misregulation in cancer. However, much less is known about protein phosphatases influence. Phosphoprotein Phosphatase 1 (PPP1) is one of the major serine/threonine protein phosphatases who has three catalytic isoforms: PPP1CA, PPP1CB, and PPP1CC. Its function is achieved by binding to regulatory subunits, known as PPP1-interacting proteins (PIPs), which may prefer a catalytic isoform. Also, some inhibitors/enhancers may exhibit isoform specificity. Here we show that, prodigiosin (PG), a molecule with anticancer properties, promotes the formation of PPP1CA-AKT complex and not of PPP1CC-MAPK complex. Both, AKT and MAPK, are wellknown PIPs from two pathways that crosstalk and regulate melanoma cells survival. In addition, the analysis performed using surface plasmon resonance (SPR) technology indicates that PPP1

\footnotetext{
${ }^{*}$ Corresponding author.
}

How to cite this paper: Fardilha, M., et al. (2014) Phosphoprotein Phosphatase 1 Isoforms Alpha and Gamma Respond Differently to Prodigiosin Treatment and Present Alternative Kinase Targets in Melanoma Cells. Journal of Biophysical Chemistry, 5, 67-77. http://dx.doi.org/10.4236/jbpc.2014.52008 
interacts with obatoclax (OBX), a drug that belongs to the same family of PG. Overall, these results suggest that PG might, at least in part, act through PPP1C/PIPs. Also, this study is pioneer in demonstrating PPP1 isoform-specific modulation by small molecules.

\title{
Keywords
}

\author{
Phosphoprotein Phosphatase 1 Catalytic Subunit, Surface Plasmon Resonance, Mitogen-Activated \\ Protein Kinase, V-Akt Murine Thymoma Viral Oncogene, Glycogen Synthase Kinase 3
}

\section{Introduction}

Reversible phosphorylation of proteins is a major intracellular regulatory mechanism in eukaryotes, resulting from the dynamic and coordinated action of protein kinases and phosphatases [1]. This post-translational modification has been associated with many cell signaling pathways which, in turn, regulate several cellular events [2] [3]. So, unsurprisingly, some of the most prevalent pathological conditions (e.g. cancer, diabetes, and neurodegenerative conditions) are, at least in part, consequence of the abnormal phosphorylation of key proteins [4].

Phosphoprotein Phosphatase 1 (PPP1), one of the most important serine/threonine phosphatases, has been receiving increasing attention. PPP1 actions are dependent on the interaction of its catalytic subunit (PPP1C) with regulatory subunits, known as PPP1-interacting proteins (PIPs). Hence, besides the PPP1 role in cell cycle progression [5] [6] and apoptosis [7] [8], two cellular events that when dysfunctional induce tumorigenesis, it also interacts directly with proteins involved in specific types of cancer, such as BRAC1 (breast cancer) [9], AKT and MAPK (melanoma, colon cancer, etc.) [10]-[14]. These interactions suggest that PPP1C/PIPs complexes are potential targets for anticancer therapy.

Compounds that directly interfere with PPP1Chave been exploited as anticancer drugs, not only as single agents but also in combination with conventional treatments. Several naturally occurring toxins, as cantharidin (CT) and its analogues, have been extensively studied but they still have many drawbacks, including severe toxic side effects [15] [16]. Alternatively, the targeting of PPP1C/PIP complexes has been demonstrated as a remarkable strategy, as PIPs are more event, tissue, and subcellular compartment specific [4]. For example, treatment of glioblastoma and prostate cancer cells with the histone deacetylases (HDACs) inhibitor, trichostatin A (TSA), disrupts the PPP1C/HDAC6 complex, resulting in increased dephosphorylation or acetylation of, for example, AKT, thus decreasing its activity [17]. Also, the formation of the PPP1-GADD34 complex is decreased in cells treated with salubrinal, a small molecule that protects the cell from ER-stress-induced apoptosis.

A number of interesting anticancer properties have also been described for a few prodiginine family members, with prodigiosin (PG) and obatoclax (OBX) being two of the most prominentones. Previous studies have demonstrated that PG proapoptotic effect in breast cancer cells is mediated by induction of the gene NAG-1. This occurs via glycogen synthase kinase-3 $\beta$ (GSK3 $\beta$ ) activity, a downstream substrate of both PI3K/AKT and MAPK pathways, which are strongly regulated by PPP1C [18]. On the other hand, OBX was described as a BH3 mimetic, targeting several anti-apoptotic Bcl-2 family members [19] that are already known PIPs [8]. However, its mechanism of action remains poorly understood. More recently, few additional protein kinases were identified as being negatively regulated by PG. Interestingly, all of them participate in PI3K/AKT/mTOR and MAPK pathways. The inhibition of such kinases, mainly mTORC1 and mTORC2 complexes, culminates in the loss of AKT phosphorylation at S473 [20].

In order to evaluate the role of PPP1C in prodiginines-mediated effect in tumorigenesis, we analyzed the action of PG in human melanoma cell lines subjected to a PPP1C inhibition treatment. Moreover, to assess PPP1C as a potential molecular target of prodiginines, we performed a real-time interaction analysis using Surface Plasmon Resonance (SPR) technology.

\section{Material and Methods}

\subsection{Drugs and Reagents}

PG (2-methyl-3-pentyl-6-methoxyprodigiosene) was provided by Dr. R. J. Schultz of the National Cancer Drug Synthesis and Chemistry Branch Chemotherapeutic Agents Repository (Bethesda, MD). OBX, a synthetic indol- 
containing prodiginine, was provided by Dr. Roberto Quesada of the University of Burgos. CT was purchased from Sigma-Aldrich (Sintra, Portugal). All stock solutions were diluted in DMSO and stored at $-20^{\circ} \mathrm{C}$.

\subsection{Cell Lines and Culture Conditions}

Human melanoma cells SK-MEL-5 were purchased from American Type Culture Collection (Barcelona, Spain). Cells were cultured in Minimum Essential Medium (MEM, Gibco, Madrid, Spain) containing GlutaMAX, supplemented with 5\% fetal bovine serum (FBS, Gibco, Madrid, Spain), $1 \%$ L-glutamine, $10 \mathrm{mg} / \mathrm{ml}$ antibiotic and antimycotic mixture (Gibco, Madrid, Spain) and non-essential amino acids, and grown in a $5 \% \mathrm{CO}_{2}$ humidified incubator at $37^{\circ} \mathrm{C}$.

\subsection{Western Blot Analysis}

Briefly, cells were treated with $10 \mu \mathrm{MCT}$ and $0.15 \mu \mathrm{M}$ or $0.38 \mu \mathrm{M}$ PG for 30 or 60 minutes (details ofthe duration of the treatments are provided on each figure). Cells were then harvested in $1 \%$ sodium dodecyl sulfate (SDS) and the protein content of the cell lysates was determined by the bicinchoninic acid method (BCA) (Pierce, Rockford, IL, USA) using bovine serum albumin as standard. Mass-normalized samples were loaded onto SDS-PAGE and subsequently transferred onto nitrocellulose membranes (GE Healthcare, Carnaxide, Portugal). Immunoblotting of the transferred proteins was performed as previously described [21] and immunoreactive bands were revealed by incubation with a HRP-conjugated secondary antibody followed by ECL detection (Amersham Pharmacia, Piscataway, USA). All the experiments were repeated at least three times.

\subsection{Antibodies}

Anti-PPP1CA antibody (CBC2C, dilution 1:2500) and anti-PPP1CC antibody (CBC3C, dilution 1:5000), against the C-terminus of the proteins, are homemade rabbit polyclonal antibodies from the Centre for Cell Biology (University of Aveiro, Portugal) [22]. Anti-AKT (cat. 9272S, dilution 1:1000), anti-phospho AKT (S473) (cat. 9271S, dilution 1:1000) and anti-phospho GSK3 $\alpha / \beta$ (S21/9) (cat. 9331S), all raised in rabbit, were purchased from Cell Signalling (Massachusetts, USA). Mouse anti-MAPK antibody (cat. 612280, dilution 1:1000) was acquired from BD Biosciences (Madrid, Spain), and rabbit anti-phospho MAPK (Thr202/Tyr204) (dilution 1:1000) antibody was obtained from Santa Cruz Biotechnology, Inc. (Heidelberg, Germany). Monoclonal antiPARP antibody and anti- $\beta$ tubulin (dilution 1:1000), both raised in mouse, were purchased from Zymed Laboratories, Inc. (Cambridge, UK). Goat anti-actin antibody (cat. sc-1616) was obtained from Santa Cruz Biotechnology, Inc. (Heidelberg, Germany), and mouse anti-vinculinantibody (cat. V9131) from Sigma-Aldrich (Sintra, Portugal).

\subsection{Surface Plasmon Resonance Assays}

Surface plasmon resonance (SPR) was performed using Biacore T100, which takes advantage of SPR detection at gold surfaces and reagent handling in a microfluidic system. This sensitive and high-performance system enable monitoring in real time, using label-free assay formats, the interaction between biomolecules. PPP1CA (New England BioLabs, Hitchin, UK) was covalently attached to a CM5 sensor chip according to amine coupling protocol. Activation of the surface with 1-ethyl-3 (3-dimethylaminopropyl) carbodiimide (EDC) and N-hydroxysuccinimide (NHS) was followed by injection ofPPP1CA protein; the excess of reactive groups were deactivated with ethanolamine. PPP1CA was diluted to $1 \mathrm{ng} / \mu \mathrm{l}$ in acetate buffer $\mathrm{pH} 4.8$, and immobilized to a level of 3982 response units (RU). All the assays were carried out at $25^{\circ} \mathrm{C}$ using HBSN buffer (10 mM Hepes and 150 $\mathrm{mM} \mathrm{NaCl}, \mathrm{pH}$ 7.4) supplemented with $100 \mathrm{mM} \mathrm{MgCl}_{2}$. The same buffer was used to dilute OBX. OBX was injected over sensor surfaces for $60 \mathrm{~s}$ at $30 \mu \mathrm{l} / \mathrm{min}$. The complexes were regenerated back using $25 \mathrm{mM} \mathrm{NaOH}$. The same protocol was followed for PPP1CC, a homemade purified protein, except for the running buffer whose constitution was previously described in Stenlund, P. et al. [23].

\section{Results}

\subsection{Prodigisin and Cantharidin Treatment Have Opposite Effects Onphosphorylated AKT in Melanoma Cells}

In a first step, we aimed to determine the involvement of PPP1 in the reversible phosphorylation system that re- 
gulates the AKT signaling pathway. For this, we determined the expression levels of phosphorylated AKT (pAKT, S463) in human melanoma cells treated with PG or CT. SK-MEL-5 cells, derived from axillary node metastasis were treated with $1 \mu \mathrm{M}$ PG (corresponding to the IC50 at 24 hours [20]) or $10 \mu \mathrm{M}$ CT (a concentration that inhibits PPP1 [24]) for 30 and 60 minutes. Treatment with PG decreased pAKT expression levels by $60 \%$ after 30 minutes and by $80 \%$ after 60 minutes treatment, which is in accordance with previous studies [20]. By contrast, CT treatment increased pAKT by $60 \%$ after 30 minutes, with levels returning to near the baseline after 60 minutes (Figure 1).

\subsection{PPP1 Binds Prodiginines Family Members}

Taking into account the previous results, we hypothesized that PG could be acting through PPP1. Thus, we used a real-time interaction approach, based on SPR technology, to address the ability of prodiginines family members to bind PPP1. This technique was previously applied to characterize the binding of prodiginines to mTOR [20].

Binding experiments were initiated by immobilizing purified recombinant proteins PPP1CA or PPP1CC on a sensor surface. Then, the analytes (PG and OBX) were injected in solution over the surface and the interactions were analyzed, providing kinetics and affinity data in the nanomolar range. Due to technical constrains, including PG precipitation in the buffer used, we were not allowed to achieve accurate conclusions about PPP1CA/CC interaction with PG. On the other hand, predicted affinity and kinetic constants showed that OBX binded to the tested isoform, PPP1CA, with a calculated $\mathrm{K}_{\mathrm{D}}$ in a range of nanomolar $(1.8 \mathrm{E}-10 \pm 1.1 \mathrm{E}-10 \mathrm{M})$. The KD obtained indicates a high affinity interaction. Moreover, the disassociation constant Kd (0.017 s-1) indicates that OBX binds to PPP1CA, forming a stable complex (Figure 2).

In what concerns PPP1CC, we performed the experiments according to Stenlund, P. et al. [25], but we used calyculin A instead of okadaic acid in the coupling buffer. However, results were not as consistent as for PPP1CA, showing only a minorbind capability for OBX, along with a low specificity interaction (data not shown).

\subsection{Cantharidin and Prodigiosin Actions on PPP1C Areisoform-Specific}

To address the effects of PG and CT on PPP1CA and PPP1CC, we performed a sequential treatment on cultured cells. SK-MEL-5 cells were pre-incubated for 30 minutes with $10 \mu \mathrm{M}$ CT followed by incubation with $0.15 \mu \mathrm{M}$ and $0.38 \mu \mathrm{M}$ PG (corresponding to the IC12.5 and IC25 at 24 hours, respectively) for 30 minutes or 60 minutes. The expression levels of both isoforms were then assessed (Figure 3(a) and Figure 3(b)).

To assure that the observed effect was exclusively the result of the action of the drugs and not because of cell death we analyzed apoptosis using the advanced apoptosis marker PARP. For that, we evaluated the ratio of cleaved PARP/total PARP (Figure 3(c)).

PPP1CA and PPP1CC did not exhibited similar responses to PG treatment preceded by CT treatment. A treatment of $0.15 \mu \mathrm{M}$ PG for 30 minutes decreased PPP1CA levels by 25\%, while increased PPP1CC levels in $80 \%$. After this initial increase, PPP1CC levels decreased along with the augment of PG concentration and incubation time. On the other hand, the decrease in PPP1CA levels remained observable followed by a 60 minutes PG treatment, but when treated with $1 \mu \mathrm{M}$ PG PPP1CA levels increased.

\subsection{PPP1CA and PPP1CC Act on Different Signaling Pathways in Response to Prodigiosin: AKT and MAPK Pathways}

The explanation for the different responses of PPP1 isoforms to PG treatment, in human melanoma cells, may lie on distinct downstream effects on particular genes, which are targets of relevant signaling proteins. As AKT phosphorylation is affected by PG treatment, we analyzed changes on AKT and MAPK pathways in response tothe referred treatment, and addressed if the results correlate with PPP1CA and PPP1CC expression levels.

Interestingly, the ratio pAKT/AKT varied inversely to PPP1CA levels (Figure 3(a) and Figure 4(a)), whereas MAPK/pMAPK changed in accordance to PPP1CC levels (Figure 3(b) and Figure 4(b)). After 30 minutes of PG treatment, PPP1CCachieved its highest expression level, corresponding to the lowest level of pMAPK/MAPK, with the opposite being observed after 60 minutes of PG treatment. In addition, the expression pattern of GSK3, a common substrate of AKT and MAPK, and its phosphorylated form, pGSK3, were analyzed 


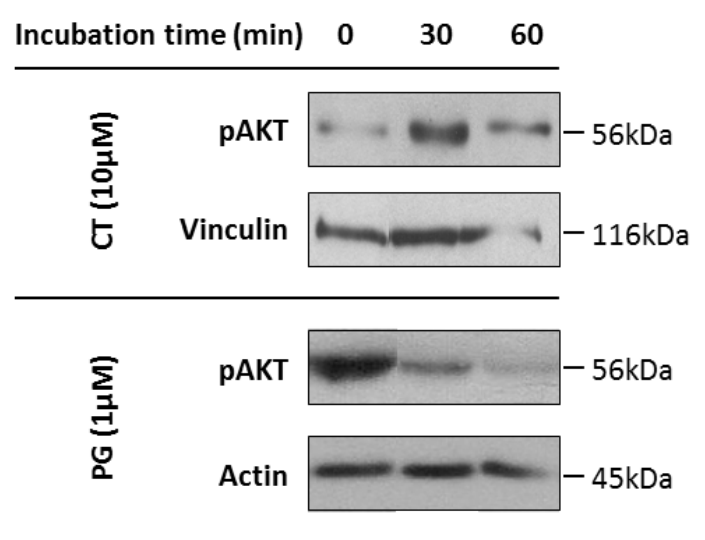

Figure 1. Expression of phosphorylated v-Akt murine thymoma viral oncogene(pAKT)in SK-MEL-5 cells treated with prodigiosin (PG) or cantharidin (CT). Cells were treated with $1 \mu \mathrm{M}$ PG or $10 \mu \mathrm{M} \mathrm{CT}$ and cell extracts were assayed for pAKT expression by immunoblotting. Actin and vinculin were used as loading controls. Experiments were repeated at least twice.

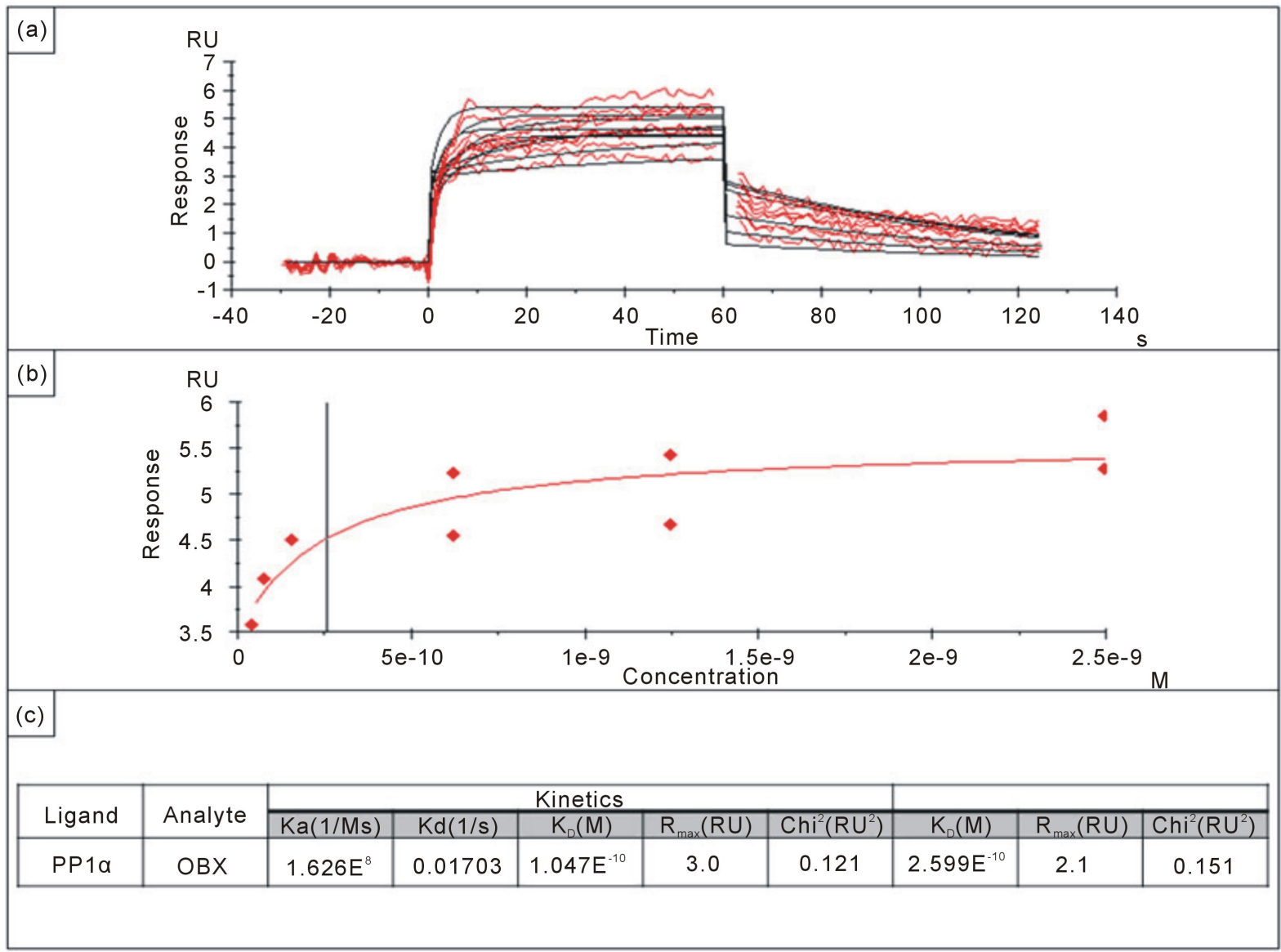

Figure 2. Kinetic and affinity characterization of obatoclax binding to phosphoprotein phosphatase 1 isoform $\alpha$ (PPP1CA) by surface plasmon resonance assay. Obatoclax (OBX) was injected, at a range of concentrations between $0.04 \mathrm{nM}$ and 2.5 $\mathrm{nM}$, over the immobilized PPP1CA. Sensograms and curve fits (smooth lines), assuming 1:1 interactions, are depicted in panel (a) and (b). Reference and blank data were subtracted. The similar dissociation rates (KD) on both kinetic and affinity sensograms (panel (c)) confirms the specificity of the binding.Experiments were repeated at least twice. 


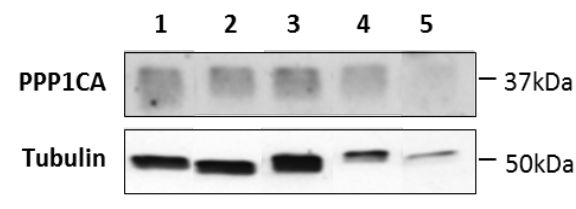

(a)

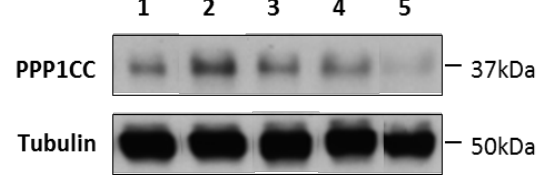

(b)

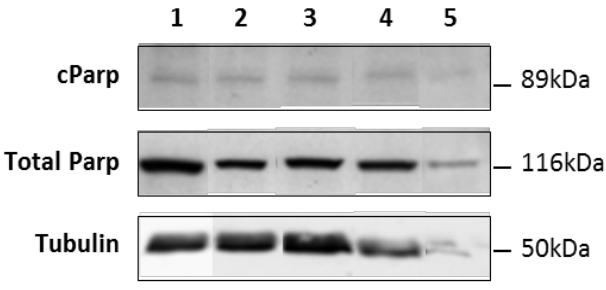

(c)

Figure 3. Phosphoprotein phosphatase 1 isoforms $\alpha$ and $\gamma$ (PPP1CA and PPP1CC, respectively) have different responses to prodigiosin (PG) treatment in human melanoma cells. SK-MEL-5 cells were treated with $10 \mu \mathrm{M}$ cantharidin (CT) for 30 minutes and then were incubated with $0.15 \mu \mathrm{M}$ or $0.38 \mu \mathrm{M}$ PG for 30 minutes (2 and 4 ) or 60 minutes ( 3 and 5 ). As control (1), cells were incubated for 90 minutes with $10 \mu \mathrm{M} \mathrm{CT}$. Cell extracts were assayed for PPP1CA (a) and PPP1CC (b) expression by immunoblotting. Apoptosis was assed using total poly ADP ribose polymerase (PARP) and cleaved PARP (cPARP). Tubulin was used as loading control. Experiments were repeated at least twice.

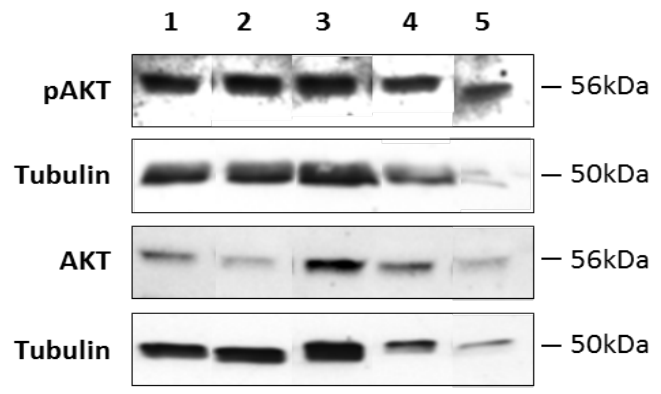

(a)

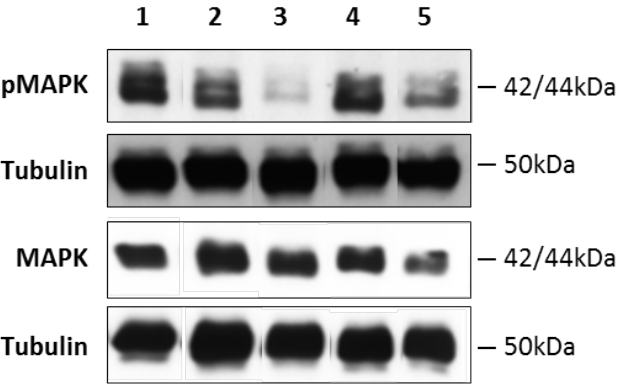

(b)

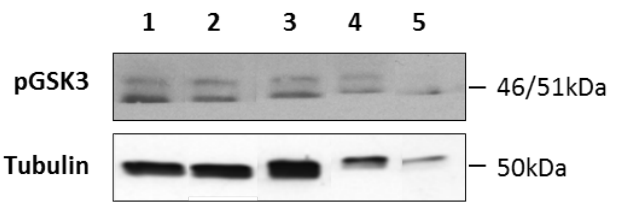

(c)

Figure 4. Phosphoprotein phosphatase 1 isoforms $\alpha$ and $\gamma$ (PPP1CA and PPP1CC, respectively) act on kinases from different signaling pathways. Cells were treated with $10 \mu \mathrm{M}$ cantharidin (CT) for 30 minutes, after which were incubated with $0.15 \mu \mathrm{M}$ or $0.38 \mu \mathrm{M}$ prodigiosin (PG) for 30 minutes (2 and 4) or 60 minutes (3 and 5). As control (1), cells were incubated for 90 minutes with $10 \mu \mathrm{M} \mathrm{CT}$. Cell extracts were assayed by immunoblotting for v-Akt murine thymoma viral oncogene (AKT) and phosphorylated AKT (pAKT) (a), mitogen-activated protein kinase (MAPK) and phosphorylated MAPK (pMAPK) (b), and glycogen synthase kinase-3 beta (GSK3) (c). Tubulin was used as loading control. Experiments were repeated at least twice.

under the same conditions (Figure 4(c)). Results show that pGSK3 expression levels decreased along with the decreased of pMAPK (the active form).

\section{Discussion}

Reversible protein phosphorylation is an essential post-translational modification that regulates numerous cellu- 
lar processes. The impairment of the correct balance between protein kinases and phosphatases leads to the onset and progression of several pathological conditions, including cancer [6].

Protein kinases have been shown to be dysfunctional in many types of cancer; therefore, kinase inhibitors have been largely explored for therapeutic purposes [26] [27]. Protein phosphatases, on the other hand, remain poorly understood but have been receiving increasing attention. PPP1C, a major serine/threonine phosphatase whose activity is dependent on the partners it binds to, has been extensively studied as a target for pharmacological intervention. A treatment used in glioblastoma and in prostate cancer cells targets the complex PPP1C/ HDAC6, which induces the dephosphorylation of pAKT — component of a signaling pathway that, when activated, induces cell proliferation and survival [28]. Previous work from Xiao, L., et al. and Thayyullathil, F., et al. showed that PPP1C could bind pAKT, being responsible for its dephosphorylation at T450 and S473 residues [11] [12]. On the other hand, work from Soto-Cerrato, V., et al. stated that PG, a molecule with anticancer properties, also promotes the dephosphorylation of pAKT [18]. These assumptions led us to hypothesize that PG could be acting, at least in part, through PPP1C/PIPs complexes.

Here, we addressed the effect of PPP1 Cinhibition in the expression levels of pAKT (S473), using human melanoma cells where the AKT pathway has been shown to be hyperactivated [29]. Indeed, the levels of pAKT (S473) increased after 30 minutes of CT treatment and decreased in response to PG treatment as expected (Figure 1). This indicates that AKT dephosphorylation, might be, at least in part, accomplished by PPP1, as already observed in other studies [30]. The mechanism by which PG leads to the dephosphorylation of AKT, nonetheless, remains unknown.

In a next step, to analyze the binding ability of PG to PPP1C, we first performed an in vitro binding assay using SPR. Unfortunately, PG precipitated very easily during the experimental procedure and OBX (an analogue from the same family) was used instead. Overall, SPR analysis results suggest that PPP1 is one of the targets of prodiginines, but isoforms exhibit distinct affinities for the drug. Affinity and kinetics evaluation of the interaction confirmed the specificity and high affinity of the bindingtoPPP1CA (Figure 2(a) and Figure 2(b)). Previously, a similar assay was performed to test the binding between PPP1CCA and two PPP1C inhibitors (CT and okadaic acid) [23]. Although the immobilization conditions and buffers were different, we can draw some putative interesting conclusions that, nevertheless, have to be proved in a near future. The $K_{d}$ for PPP1CA/OBX binding was higher (Figure 2(c)) than for PPP1CA/CT, thus OBX could hypothetically displace CT from PPP1C. However, PPP1CA/CT binding appears to be more stable because the $K_{d}$ is lower [23].

After demonstrating that PPP1CA can bind to a prodiginine family member and knowing that PPP1C inhibition enhances the phosphorylation of AKT [31], efforts were made to understand the relationship between PPP1C and PG, and their modulatory potential on relevant signaling pathways in human melanoma cells.

The CT pre-treatment for 30 minutes, as suggested by our results (Figure 3) and by others [24], should inhibit PPP1C but not change significantly its amounts. Hence, the variation in the expression levels of PPP1C must be due to the presence of PG. Interestingly, the alterations induced on the PPP1C isoforms analyzed were different, suggesting a distinct mode of action of PG on PPP1CA and PPP1CC.There is no literature concerning PPP1 isoforms-specific inhibition or enhancement by small molecules, so this data unlocks very interesting issues.

PG seems to displace CT from PPP1C (Figure 3) and, somehow, alters the amount of PPP1 within the cells. The duration of the treatment was too short to induce significant changes in protein expression at the mRNA level; therefore, the explanation may lie in the alteration of protein degradation processes and consequent promotion of the targeting of PPP1CA to degradation. In contrast, PPP1CC peaked after 30 minutes of $0.15 \mu \mathrm{MPG}$ treatment (Figure 3(b)), which may correspond to a compensatory effect resulting, for example, from PPP1CC mRNA local translation [32], and that could be bypassed through increasing the concentrations of PG or the time of incubation.

Another remarkable observation was that the expression pattern of PPP1CAcorrelated with the expression pattern of AKT (Figure 4(a)), but the same was verified for PPP1CC. For this reason, we progressed for the study of another kinase that could be a preferential target for PPP1CC. MAPK signaling pathway, which cross-talks with the AKT signaling pathway and is inhibited when the latter one is active, have also been reported as being altered in melanoma [13]. Surprisingly, the phosphorylation of MAPK (Figure 4(b)) increased when the expression levels of PPP1CCwere reduced. After 30 minutes of $0.15 \mu \mathrm{M}$ and $0.38 \mu \mathrm{MPG}$ treatment the decrease in pMAPK phosphorylation was of $40 \%$ and $90 \%$, respectively. However, this decrease was not proportional to the increase in PPP1CCexpression levels, as the increase was of $80 \%(0.15 \mu \mathrm{M})$ and $20 \%(0.38$ $\mu \mathrm{M})$. So, we hypothesized that another kinase might also be involved. In a next step we assessed GSK3 expres- 
sion levels (Figure 4(c)), a common substrate of both AKT and MAPK, and the results suggest that it is under the regulation of MAPK in the conditions of the experiment. All together, the results suggest that PPP1CA specifically dephosphorylates PAKT, while pMAPK is a preferential target for PPP1CC. Also, in the conditions of the experiments, MAPK appears to be the major kinase responsible for inactivating GSK3 by phosphorylation.

\section{Conclusions}

The identification of putative targets for pharmacological interference, mainly signaling molecules, has been interesting the research community and also pharmaceutical companies for the last decade. Recently, protein phosphatases have become also prime focus of attention, in particularPPP1/PIP complexes due to their specificity [28]. In what concerns cancer, several components of signaling pathways have shown to be deregulated and, apart from that, more than one pathway may contribute to carcinogenesis.

In melanoma, several studies have been pointing out the cross-talk between MAPK and AKT signaling pathways [13]. For instance, B-RAF activity increases after treatment with an AKT inhibitor [33] [34]. Interactions between different signaling pathways can regulate drugs mode of action, turning them more or less effective or promoting drug resistance. Several studies have shown that the cross-talk between AKT and MAPK pathways can be used more efficiently to treat melanoma by inhibiting AKT, thus promoting apoptosis and activating RAF to induce senescence [13].

Here we show that PPP1CA preferentially binds AKT, whereasPPP1CC preferential targets MAPK. In the presence of PG, PPP1CC will be targeted to degradation, increasing the phosphorylation of both MAPK and GSK3, and ultimately this will lead to senescence [13]. At the same time, PPP1CA will bind AKT, promoting its dephosphorylation and consequently cell apoptosis (Figure 5) [11] [13].

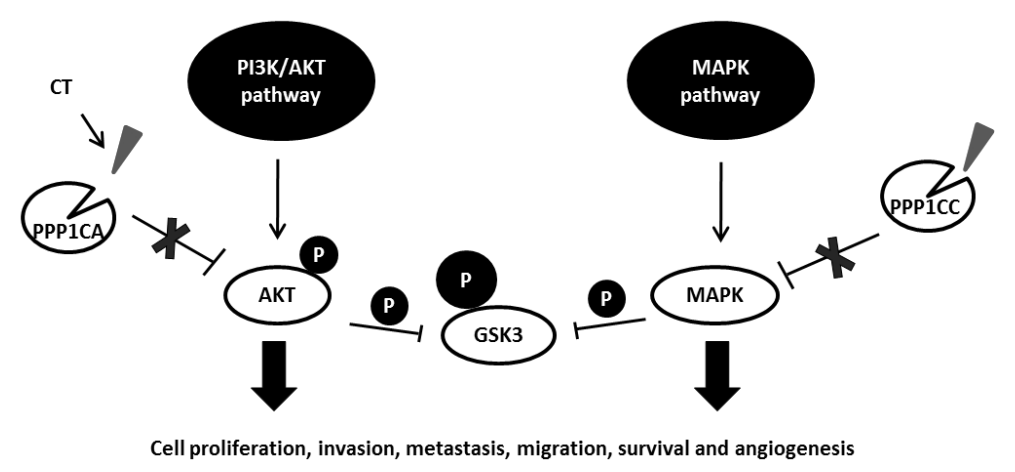

(a)

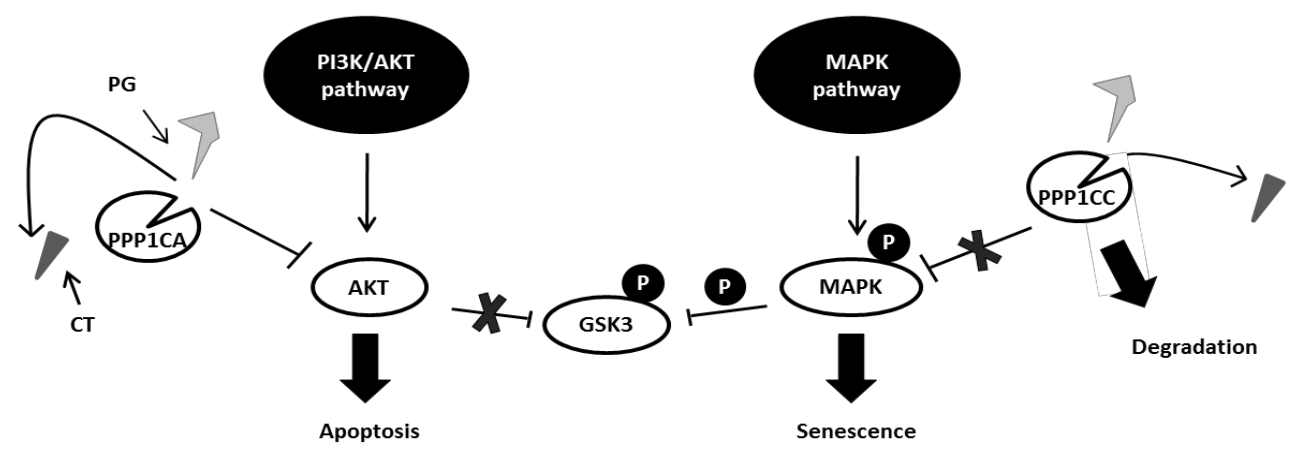

(b)

Figure 5. Model for the action of phosphoprotein phosphatase 1 isoforms $\alpha$ and $\gamma$ (PPP1CA and PPP1CC, respectively) on the v-Akt murine thymoma viral oncogene (AKT) and mitogen-activated protein kinase (MAPK) signaling pathways. Phosphoprotein phosphatase 1 isoforms, when inhibit by cantharidin (CT), promote the activation of both pathways. However, prodigiosin (PG) may be able to displace CT due its higher affinity, taking PPP1CC to degradation. To compensate, PPP1CA levels rise, thus dephosphorylating AKT and, consequently, overcoming the inhibition of Raf and promoting senescence and apoptosis. 
In conclusion, we proposed a PPP1C isoform-specific dephosphorylation of AKT and MAPK, which could be modulated by PG, hence determining melanoma cells survival and disease progression.

\section{Acknowledgements}

The authors thank Marta Taulés for technical assistance from Centres Científics i Tecnològics (Universitat de Barcelona).

\section{Funding}

This work was supported by grants from Fundação para a Ciência e Tecnologia (FCT) of the Portuguese Ministry of Science and Higher Education (PTDC/DTP-PIC/0460/2012) and by FEDER through Eixo I do Programa Operacional Fatores de Competitividade (POFC) (FCOMP-01-0124-FEDER-028692) co-funded by QREN.

\section{Author Disclosure Statement}

No competing financial interests exist.

\section{References}

[1] Shi, Y. (2009) Serine/Threonine Phosphatases: Mechanism through Structure. Cell, 139, 468-484. http://dx.doi.org/10.1016/j.cell.2009.10.006

[2] Wera, S. and Hemmings, B.A. (1995) Serine/Threonine Protein Phosphatases. Biochemical Journal, 311, 17-29.

[3] Bollen, M. (2001) Combinatorial Control of Protein Phosphatase-1. Trends in Biochemical Sciences, 26, 426-431. http://dx.doi.org/10.1016/S0968-0004(01)01836-9

[4] Fardilha, M., Esteves, S., Korrodi, L., da Cruz e Silva, O. and da Cruz e Silva, E. (2010) The Physiological Relevance of Protein Phosphatase 1 and Its Interacting Proteins to Health and Disease. Current Medicinal Chemistry, 17, 3996-4017.

[5] Ludlow, J.W. and Nelson, D.A. (1995) Control and Activity of Type-1 Serine/Threonine Protein Phosphatase during the Cell Cycle. Seminars in Cancer Biology, 6, 195-202. http://dx.doi.org/10.1006/scbi.1995.0026

[6] Hubbard, M.J. and Cohen, P. (1993) On Target with a New Mechanism for the Regulation of Protein Phosphorylation. Trends in Biochemical Sciences, 18, 172-177. http://dx.doi.org/10.1016/0968-0004(93)90109-Z

[7] Garcia, A., Cayla, X., Guergnon, J., Dessauge, F., Hospital, V., Rebollo, M.P., Fleischer, A. and Rebollo, A. (2003) Serine/Threonine Protein Phosphatases PP1 and PP2A Are Key Players in Apoptosis. Biochimie, 85, 721-726. http://dx.doi.org/10.1016/j.biochi.2003.09.004

[8] Klumpp, S. and Krieglstein, J. (2002) Serine/Threonine Protein Phosphatases in Apoptosis. Current Opinion in Pharmacology, 2, 458-462. http://dx.doi.org/10.1016/S1471-4892(02)00176-5

[9] Winter, S., Bosnoyan-Collins, L., Pinnaduwage, D. and Andrulis, I. (2007) The Interaction of PP1 with BRCA1 and Analysis of Their Expression in Breast Tumors. BMC Cancer, 7, 85. http://dx.doi.org/10.1186/1471-2407-7-85

[10] Osaki, M., Oshimura, M. and Ito, H. (2004) PI3K-Akt Pathway: Its Functions and Alterations in Human Cancer. Apoptosis, 9, 667-676. http://dx.doi.org/10.1023/B:APPT.0000045801.15585.dd

[11] Xiao, L., Gong, L.L., Yuan, D., Deng, M., Zeng, X.M., Chen, L.L., Zhang, L., Yan, Q., Liu, J.P., Hu, X.H., Sun, S.M., Liu, J., Ma, H.L., Zheng, C.B., Fu, H., Chen, P.C., Zhao, J.Q., Xie, S.S., Zou, L.J., Xiao, Y.M., Liu, W.B., Zhang, J., Liu, Y. and Li, D.W.C. (2010) Protein Phosphatase-1 Regulates Akt1 Signal Transduction Pathway to Control Gene Expression, Cell Survival and Differentiation. Cell Death and Differentiation, 17, 1448-1462. http://dx.doi.org/10.1038/cdd.2010.16

[12] Thayyullathil, F., Chathoth, S., Shahin, A., Kizhakkayil, J., Hago, A., Patel, M. and Galadari, S. (2011) Protein Phosphatase 1 Dependent Dephosphorylation of Akt Is the Prime Signaling Event in Sphingosine-Induced Apoptosis in Jurkat Cells. Journal of Cellular Biochemistry, 112, 1138-1153. http://dx.doi.org/10.1002/jcb.23033

[13] Inamdar, G.S., Madhunapantula, S.V. and Robertson, G.P. (2010) Targeting the MAPK Pathway in Melanoma: Why Some Approaches Succeed and Other Fail. Biochemical Pharmacology, 80, 624-637. http://dx.doi.org/10.1016/j.bcp.2010.04.029

[14] Quevedo, C., Salinas, M. and Alcázar, A. (2003) Initiation Factor 2B Activity Is Regulated by Protein Phosphatase 1, Which Is Activated by the Mitogen-activated Protein Kinase-dependent Pathway in Insulin-Like Growth Factor 1-Stimulated Neuronal Cells. Journal of Biological Chemistry, 278, 16579-16586. http://dx.doi.org/10.1074/jbc.M212936200 
[15] Sakoff, J.A., Ackland, S.P., Baldwin, M.L., Keane, M.A. and McCluskey, A. (2002) Anticancer Activity and Protein Phosphatase 1 and 2A Inhibition of a New Generation of Cantharidin Analogues. Investigational New Drugs, 20, 1-11. http://dx.doi.org/10.1023/A:1014460818734

[16] McCluskey, A., Ackland, S.P., Bowyer, M.C., Baldwin, M.L., Garner, J., Walkom, C.C. and Sakoff, J.A. (2003) Cantharidin Analogues: Synthesis and Evaluation of Growth Inhibition in a Panel of Selected Tumour Cell Lines. Bioorganic Chemistry, 31, 68-79. http://dx.doi.org/10.1016/S0045-2068(02)00524-2

[17] Chen, C.S., Weng, S.C., Tseng, P.H., Lin, H.P. and Chen, C.S. (2005) Histone Acetylation-Independent Effect of Histone Deacetylase Inhibitors on Akt through the Reshuffling of Protein Phosphatase 1 Complexes. Journal of Biological Chemistry, 280, 38879-38887. http://dx.doi.org/10.1074/jbc.M505733200

[18] Soto-Cerrato, V., Viñals, F., Lambert, J.R., Kelly, J.A. and Pérez-Tomás, R. (2007) Prodigiosin Induces the Proapoptotic Gene NAG-1 via Glycogen Synthase Kinase-3 $\beta$ Activity in Human Breast Cancer Cells. Molecular Cancer Therapeutics, 6, 362-369. http://dx.doi.org/10.1158/1535-7163.MCT-06-0266

[19] Perez-Tomas, R. and Vinas, M. (2010) New Insights on the Antitumoral Properties of Prodiginines. Current Medicinal Chemistry, 17, 2222-2231.

[20] Espona-Fiedler, M., Soto-Cerrato, V., Hosseini, A., Lizcano, J.M., Guallar, V., Quesada, R., Gao, T. and Pérez-Tomás, R. (2012) Identification of Dual mTORC1 and mTORC2 Inhibitors in Melanoma Cells: Prodigiosin vs. Obatoclax. Biochemical Pharmacology, 83, 489-496. http://dx.doi.org/10.1016/j.bcp.2011.11.027

[21] da Cruz e Silva, O., Fardilha, M., Henriques, A., Rebelo, S., Vieira, S. and Da Cruz e Silva, E. (2004) Signal Transduction Therapeutics. Journal of Molecular Neuroscience, 23, 123-142. http://dx.doi.org/10.1385/JMN:23:1-2:123

[22] Fardilha, M., Esteves, S.L.C., Korrodi-Gregório, L., Vintém, A.P., Domingues, S.C., Rebelo, S., Morrice, N., Cohen, P.T.W., da Cruz e Silva, O.A.B. and da Cruz e Silva, E.F. (2011) Identification of the Human Testis Protein Phosphatase 1 Interactome. Biochemical Pharmacology, 82, 1403-1415. http://dx.doi.org/10.1016/j.bcp.2011.02.018

[23] Stenlund, P., Frostell-Karlsson, A. and Karlsson, O.P. (2006) Studies of Small Molecule Interactions with Protein Phosphatases Using Biosensor Technology. Analytical Biochemistry, 353, 217-225. http://dx.doi.org/10.1016/j.ab.2006.03.004

[24] Honkanen, R.E. (1993) Cantharidin, Another Natural Toxin That Inhibits the Activity of Serine/Threonine Protein Phosphatases Types 1 and 2A. FEBS Letters, 330, 283-286. http://dx.doi.org/10.1016/0014-5793(93)80889-3

[25] Stenlund, P., Frostell-Karlsson, Å. and Karlsson, O.P. (2006) Studies of Small Molecule Interactions with Protein Phosphatases Using Biosensor Technology. Analytical Biochemistry, 353, 217-225. http://dx.doi.org/10.1016/j.ab.2006.03.004

[26] Becker, J.C., Kirkwood, J.M., Agarwala, S.S., Dummer, R., Schrama, D. and Hauschild, A. (2006) Molecularly Targeted Therapy for Melanoma. Cancer, 107, 2317-2327. http://dx.doi.org/10.1002/cncr.22273

[27] Smalley, K.S.M. and Herlyn, M. (2005) Targeting Intracellular Signaling Pathways as a Novel Strategy in Melanoma Therapeutics. Annals of the New York Academy of Sciences, 1059, 16-25. http://dx.doi.org/10.1196/annals.1339.005

[28] McConnell, J.L. and Wadzinski, B.E. (2009) Targeting Protein Serine/Threonine Phosphatases for Drug Development. Molecular Pharmacology, 75, 1249-1261. http://dx.doi.org/10.1124/mol.108.053140

[29] Stahl, J.M., Sharma, A., Cheung, M., Zimmerman, M., Cheng, J.Q., Bosenberg, M.W., Kester, M., Sandirasegarane, L. and Robertson, G.P. (2004) Deregulated Akt3 Activity Promotes Development of Malignant Melanoma. Cancer Research, 64, 7002-7010. http://dx.doi.org/10.1158/0008-5472.CAN-04-1399

[30] Thayyullathil, F., Chathoth, S., Shahin, A., Kizhakkayil, J., Hago, A., Patel, M. and Galadari, S. (2011) Protein Phosphatase 1-Dependent Dephosphorylation of Akt Is the Prime Signaling Event in Sphingosine-Induced Apoptosis in Jurkat Cells. Journal of Cellular Biochemistry, 112, 1138-1153. http://dx.doi.org/10.1002/jcb.23033

[31] Li, L., Ren, C.H., Tahir, S.A., Ren, C. and Thompson, T.C. (2003) Caveolin-1 Maintains Activated Akt in Prostate Cancer Cells through Scaffolding Domain Binding Site Interactions with and inhibition of Serine/Threonine Protein Phosphatases PP1 and PP2A. Molecular and Cellular Biology, 23, 9389-9404. http://dx.doi.org/10.1128/MCB.23.24.9389-9404.2003

[32] Warner, S.L., Bearss, D.J., Han, H. and Von Hoff, D.D. (2003) Targeting Aurora-2 Kinase in Cancer1. Molecular Cancer Therapeutics, 2, 589-595.

[33] Merighi, S., Benini, A., Mirandola, P., Gessi, S., Varani, K., Leung, E., Maclennan, S., Baraldi, P.G. and Borea, P.A. (2006) Modulation of the Akt/Ras/Raf/MEK/ERK pathway by $A_{3}$ Adenosine Receptor. Purinergic Signalling, 2, 627 632. http://dx.doi.org/10.1007/s11302-006-9020-4

[34] Guan, K.L., Figueroa, C., Brtva, T.R., Zhu, T., Taylor, J., Barber, T.D. and Vojtek, A.B. (2000) Negative Regulation of the Serine/Threonine Kinase B-Raf by Akt. Journal of Biological Chemistry, 275, 27354-27359. 


\section{Abbreviations}

AKT: v-Akt murine thymoma viral oncogene;

Bcl-2: B-cell lymphoma 2;

BRAC1: breast cancer 1 early onset;

B-RAF: v-raf murine sarcoma viral oncogene homolog B;

CT: cantharidin;

ER: endoplasmic reticulum;

GADD34: growth arrest and DNA damage-inducible protein 34;

GSK3 $\beta$ : glycogen synthase kinase-3 beta;

HDAC: histone deacetylase;

MAPK: mitogen-activated protein kinase;

mTOR: mammalian target of rapamycin;

mTORC: mTOR complex;

NAG-1: nonsteroidal anti-inflammatory drug-activated gene;

OBX: obatoclax;

PARP: poly ADP ribose polymerase;

PI3K: phosphoinositide 3-kinase;

PIP: PPP1-interacting protein;

PG: prodigiosin;

PPP1: phosphoprotein phosphatase 1;

PPP1C: phosphoprotein phosphatase 1 catalytic subunit;

PPP1CA: phosphoprotein phosphatase 1 isoform $\alpha$;

PPP1CC: phosphoprotein phosphatase 1 isoform $\gamma$;

SPR: surface plasmon resonance;

TSA: trichostatin A. 